\title{
The Approximate Solutions of Fredholm Integrodifferential-Difference Equations with Variable Coefficients via Homotopy Analysis Method
}

\author{
Seydi Battal Gazi Karakoç, Aytekin Eryılmaz, and Musa Başbük \\ Department of Mathematics, Nevşehir University, 50300 Nevşehir, Turkey \\ Correspondence should be addressed to Aytekin Eryılmaz; eryilmazaytekin@gmail.com
}

Received 8 January 2013; Accepted 25 February 2013

Academic Editor: Safa Bozkurt Coskun

Copyright (c) 2013 Seydi Battal Gazi Karakoç et al. This is an open access article distributed under the Creative Commons Attribution License, which permits unrestricted use, distribution, and reproduction in any medium, provided the original work is properly cited.

\begin{abstract}
Numerical solutions of linear and nonlinear integrodifferential-difference equations are presented using homotopy analysis method. The aim of the paper is to present an efficient numerical procedure for solving linear and nonlinear integrodifferentialdifference equations. The reliability and efficiency of the proposed scheme are demonstrated by some numerical experiments and performed on the computer algebraic system.
\end{abstract}

\section{Introduction}

Currently, considerable interest in mixed integrodifferentialdifference equations (IDDEs) has been stimulated due to their numerous applications in the areas of engineering, mechanics, physics, chemistry, astronomy, biology, economics, potential theory, and electrostatics [1-7]. In integrodifferential-difference equations, the unknown function appears to be under integration sign, and it may also include the derivatives and functional arguments of the unknown function. This type of equations can be grouped into Fredholm integrodifferential-difference equations and Volterra integrodifferential-difference equations. The upper bound of the integral part of Volterra type is variable, while it is a fixed number for that of Fredholm type [1]. Since IDDEs are usually difficult to solve in an analytical manner, or to obtain closed form solution, a numerical method is needed. Many numerical methods were applied such as Taylor matrix method [2], Chebyshev finite difference method [8], Legendre tau method [4], Bessel matrix method [5], and variational iteration method [9].

In this paper, we propose an efficient method, namely homotopy analysis method, to obtain the numerical solution of $m$ th-order linear Fredholm integrodifferential-difference equation with variable coefficients. Consider

$$
\begin{aligned}
& \sum_{k=0}^{m} P_{k}(x) y^{(k)}(x)+\sum_{r=0}^{n} P_{r}^{*}(x) y^{(r)}(x-\tau) \\
& =f(x)+\int_{a}^{b} K(x, t) y(x-\tau) d t, \quad \tau \geq 0,
\end{aligned}
$$

with mixed conditions

$$
\begin{aligned}
\sum_{k=0}^{m-1}\left[a_{i k} y^{(k)}(a)+b_{i k} y^{(k)}(b)+c_{i k} y^{(k)}(c)\right] & =\lambda_{i}, \\
i=0,1, \ldots, m-1, \quad a & \leq c \leq b,
\end{aligned}
$$

where $P_{k}(x), P_{r}^{*}(x), K(x, t)$, and $f(x)$ are functions defined on $a \leq c \leq b$; the real coefficients $a_{\{i k\}}, b_{\{i k\}}, c_{\{i k\}}$, and $\lambda_{i}$ are appropriate constants.

Homotopy analysis method (HAM) was first introduced by Liao [10] to obtain series solutions of various linear and nonlinear problems. HAM is a promising method that gives us acceptable analytical results with convenient convergence [10]. In contrast to perturbation techniques, this approach is independent of any small parameters. In addition, different from all other analytic techniques, HAM provides us with a simple procedure to obtain the convergence of series of solutions, so that one can obtain accurate enough approximations by auxiliary convergence-controller parameter $\hbar$. In 
his papers, Liao used this method to solve many nonlinear problems. In [10], especially, he pointed out the basic ideas of the HAM and gave the details in theory and he has successfully applied it to many nonlinear problems [1015]. Recently, this technique has successfully been applied to several nonlinear problems, such as the viscous flows of non-Newtonian fluids [16, 17], nonlinear heat transfer [18], nonlinear Fredholm integral equations [19], the KdVtype equations [20], differential-difference equations [21], time-dependent Emden-Fowler-type equations [22], Laplace equation with Dirichlet and Neumann conditions [23], and pantograph equations [24].

\section{Homotopy Analysis Method}

The purpose of the study is to apply the homotopy analysis method to the integrodifferential-difference equation. Now suppose that $u_{0}(x)$ denotes an initial approximation guess of exact solution of $u(x)$. The auxiliary parameter $\hbar \neq 0$, which is a convergence-controller parameter, the auxiliary function $H(x) \neq 0$, and the auxiliary linear operator $L$ play important roles within the homotopy analysis method to adjust and control the convergence region of solution series. Liao constructs, using $q \in[0,1]$ as an embedding parameter, the so-called zero-order deformation equation

$$
(1-q) L\left[\phi(x ; q)-u_{0}(x)\right]=q \hbar H(x) N[\phi(x ; q)],
$$

where $\phi(x ; q)$ is unknown function to be determined and $N[\phi(x ; q)]$ is given by

$$
\begin{aligned}
N[\phi(x ; q)]= & \sum_{k=0}^{m} P_{k}(x) y^{(k)}(x)+\sum_{r=0}^{n} P_{r}^{*}(x) y^{(r)}(x-\tau) \\
& -f(x)-\int_{a}^{b} K(x, t) y(x-\tau) d t .
\end{aligned}
$$

When $q=0$, the zero-order deformation equation (3) becomes $\phi(x ; 0)=u_{0}(x)$, and when $q=1$, then the zeroorder deformation equation (3) becomes $L[\phi(x ; 1)]=0$. As $q$ increases from 0 to 1 , the solution $\phi(x ; q)$ varies from the initial guess $u_{0}(x)$ to the solution $u(x)$. So $\phi(x ; 1)$ is exactly the solution of nonlinear equation (1). Expanding $\phi(x ; q)$ in Taylor series with respect to $q$, we have

$$
\phi(x ; q)=u_{0}(x)+\sum_{m=1}^{\infty} q^{m} u_{m}(x)
$$

where

$$
u_{m}(x)=\left.\frac{1}{m !} \frac{\partial^{m} \phi(x ; q)}{\partial q^{m}}\right|_{q=0}, \quad m=0,1,2, \ldots
$$

If the auxiliary linear operator, the initial guess, the auxiliary parameter $\hbar$ which will be determined, later and the auxiliary function are properly chosen, the power series of $\phi(x ; q)$ converges at $q=1$, then the following series solution can be obtained:

$$
u(x)=u_{0}(x)+\sum_{m=1}^{\infty} u_{m}(x)
$$

where the terms $u_{m}(x)$ can be determined by the so-called high-order deformation equations. Now the vector is defined as $\vec{u}_{m}(x)=\left\{u_{0}(x), u_{1}(x), \ldots, u_{m}(x)\right\}$, and differentiating (3) equation $m$ times with respect to embedding parameter $q$, then setting $q=0$, and finally dividing by $m$ !, we obtain the so-called $m$ th-order deformation equation in the following form:

$$
L\left[u_{m}(x)-\chi_{m} u_{m-1}(x)\right]=\hbar H(x) \mathfrak{R}_{m}\left(u_{m-1}, x\right),
$$

where

$$
\begin{gathered}
\chi_{m}= \begin{cases}0, & m \leq 1, \\
1, & m>1,\end{cases} \\
\mathfrak{R}_{m}\left(u_{m-1}, x\right)=\left.\frac{1}{(m-1) !} \frac{\partial^{m-1} N[\phi(x ; q)]}{\partial q^{m-1}}\right|_{q=0} .
\end{gathered}
$$

In order to obey both the first rule of solution expression and the rule of the coefficient ergodicity, the corresponding auxiliary function is determined uniquely by $H(x)=1$ [10].

For any given nonlinear operator $N$ and the term $\mathfrak{R}_{m}\left(u_{m-1}, x\right)$ can be easily expressed by (10). So, we can obtain $u_{1}(x), u_{2}(x), \ldots$ by means of solving the linear high-order deformation equation (8). The $m$ th-order approximation of $u(x)$ is given by

$$
U_{M}(x) \cong u(x)=\sum_{m=0}^{\infty} u_{m}(x) .
$$

The foregoing approximate solution consists of $\hbar$, which is a cornerstone of the HAM in determining convergence of series solution rapidly. We may adjust and control the convergence region and rate of the solution series (11) by means of the auxiliary parameter $\hbar$. To obtain valid region of $\hbar$, we first plot the so-called $\hbar$ curves of $U^{\prime}(\alpha), U^{\prime \prime}(\alpha)$, and $U^{\prime \prime \prime}(\alpha)$, where $\alpha \in[a, b]$ and so on. According to these $\hbar$ curves, it is easy to discover the valid region of $\hbar$, which corresponds to the line segments nearly parallel to the horizontal axis.

Theorem 1 (convergence theorem). As long as the series (8) converges to $u(x)$, where $u_{m}(x)$ is governed by the high-order deformation equation (9) under the definitions (10) and (11), it must be the exact solution of (3).

\section{Numerical Examples and Error Analysis}

In the present paper, the HAM technique is applied to the integrodifferential-difference equations, and how one can control the convergence of approximate solution and make the convergence fast is shown. The method is applied to the following problems.

Example 2. Firstly, let us consider the second-order integrodifferential-difference equation with variable coefficients [4]

$$
\begin{aligned}
u^{\prime \prime} & (x)+x u^{\prime}(x)+x u(x)+u^{\prime}(x-1)+u(x-1) \\
& =e^{-x}+e+\int_{-1}^{0} t u(t-1) d t
\end{aligned}
$$


with the exact solution $u(x)=e^{-x}$ and initial conditions

$$
u(0)=1, \quad u^{\prime}(0)=-1
$$

To solve (12) by means of homotopy analysis method, we choose $u_{0}(x)=-x+1$ as initial approximation which satisfies (13) and let us define $N[\phi(x ; q)]$ as follows:

$$
\begin{aligned}
N[\phi(x ; q)]= & \phi^{\prime \prime}(x)+x \phi^{\prime}(x)+x \phi(x)+\phi^{\prime}(x-1) \\
& +\phi(x-1)-\left(1-\chi_{m}\right)\left(e^{-x}+e\right) \\
& -\int_{-1}^{0} t u(t-1) d t .
\end{aligned}
$$

Hence, the high-order deformation equation (8)-(10) is as follows:

$$
\begin{aligned}
u_{m}(x)= & \chi_{m} u_{m-1}(x)+\hbar \int_{0}^{x} \int_{0}^{\zeta} u^{\prime \prime}(\tau)+\tau u^{\prime}(\tau)+\tau u(\tau) \\
& +u^{\prime}(\tau-1)+u(\tau-1)-\left(1-\chi_{m}\right)\left(e^{-\tau}+e\right) \\
& -\int_{-1}^{0} t u(t-1) d t d \tau d \zeta .
\end{aligned}
$$

Therefore, by starting with $u_{0}(x)=-x+1$, we successively obtain $u_{i}(x), i=1,2,3, \ldots$, by (15),

$$
\begin{aligned}
u_{1}(x)= & \hbar-\hbar x-\frac{7 \hbar x^{2}}{6}-\frac{e \hbar x^{2}}{2}-\frac{\hbar x^{3}}{6}-\frac{\hbar x^{4}}{12} \\
& -\hbar \cosh (x)+\hbar \sinh (x)
\end{aligned}
$$

In this way we can get

$$
\begin{aligned}
U_{m}(x, \hbar) & =\sum_{n=0}^{m} u_{n}(x) \\
& =u_{0}(x)+u_{1}(x)+u_{2}(x)+\cdots+u_{m}(x)
\end{aligned}
$$

by choosing proper convergence parameter $\hbar$ with $m=20$. To find the valid region of $\hbar$ on the convergence of $U_{m}(x, \hbar)$ as $m$ goes to infinity, we plot the $\hbar$ curves of $U_{20}^{\prime}(1, \hbar)$ and $U_{20}^{\prime \prime}(1, \hbar)$ as shown in Figure 1 which clearly indicates that the valid region of $\hbar$ is about $-0,6<\hbar<-0,15$.

Some numerical results of $U_{20}(x, \hbar), U_{15}(x, \hbar)$, and $U_{10}(x, \hbar)$ for $\hbar=-0,368$ are presented in Table 1 , and the absolute errors of $U_{20}(x, \hbar), U_{15}(x, \hbar)$, and $U_{10}(x, \hbar)$ for $\hbar$ $=-0,368$ are given in Table 2 . We also give the graphs of the exact solution and 20th order approximation for $\hbar=$ $-0,368$ in Figure 2 and the absolute error of the 20th order approximation for $\hbar=-0,368$ in Figure 3 .

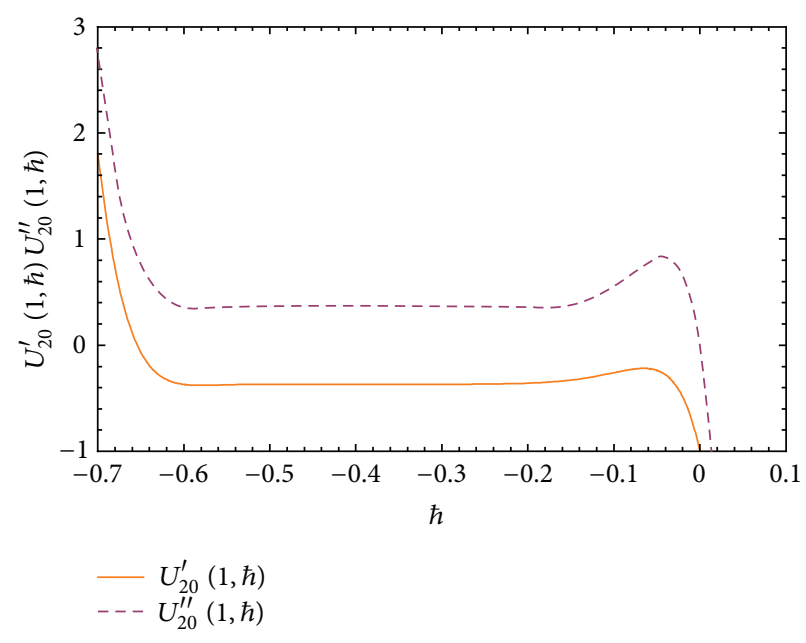

Figure 1: The $\hbar$ curves of $U_{20}^{\prime}(1, \hbar)$ and $U_{20}^{\prime \prime}(1, \hbar)$.

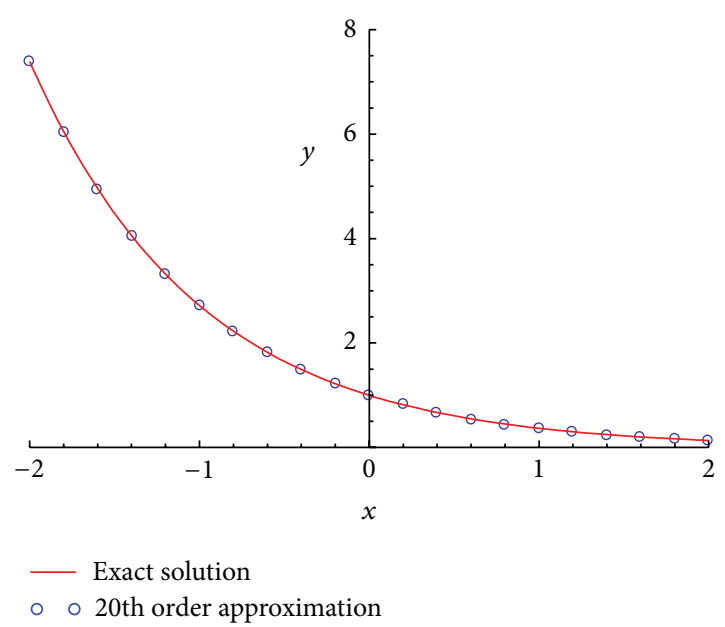

FIGURE 2: Exact solution and 20th order approximation for $\hbar=$ $-0,368$.

TABLE 1: Comparison of the exact solution and approximations of (12).

\begin{tabular}{lcccc}
\hline$x$ & $U_{\text {exact }}(x)$ & $U_{20}(x, \hbar)$ & $U_{15}(x, \hbar)$ & $U_{10}(x, \hbar)$ \\
\hline-1.0 & 2.7182818 & 2.7182784 & 2.7182076 & 2.7163599 \\
-0.8 & 2.2255409 & 2.2255388 & 1.8221033 & 1.8216473 \\
-0.6 & 1.8221188 & 1.8221182 & 1.8221033 & 1.8216473 \\
-0.4 & 1.4918246 & 1.4918250 & 1.4918242 & 1.4916964 \\
-0.2 & 1.2214027 & 1.2214031 & 1.2214053 & 1.2213995 \\
0.0 & 1.0000000 & 1.0000000 & 1.0000000 & 1.0000000 \\
\hline
\end{tabular}

Example 3. Now, consider the second-order integrodifferential-difference equation with variable coefficients [1]

$$
\begin{gathered}
u^{\prime \prime \prime}(x)-(x-1) u^{\prime \prime}(x)+(x-1) u^{\prime}(x)+u^{\prime}(x-1)-u(x) \\
=e^{x-1}+e x^{2}-\frac{1}{e} x^{2}-\frac{2}{e} x+\int_{-1}^{1}\left(x t-x^{2}\right) u(t) d t
\end{gathered}
$$


TABLE 2: The absolute errors approximations of (12).

\begin{tabular}{lccc}
\hline$x$ & Error $_{20}(x, \hbar)$ & Error $_{15}(x, \hbar)$ & Error $_{10}(x, \hbar)$ \\
\hline-1.0 & $3.4211835 E-6$ & 0.000074218443 & 0.0019219267 \\
-0.8 & $2.0579067 E-6$ & 0.000040710174 & 0.0010634492 \\
-0.6 & $5.8659108 E-7$ & 0.000015492072 & 0.00047149553 \\
-0.4 & $3.8315368 E-7$ & $4.4056641 E-7$ & 0.00012819928 \\
-0.2 & $3.8067782 E-7$ & $2.5785269 E-6$ & $3.2519805 E-6$ \\
0.0 & 0 & 0 & 0 \\
\hline
\end{tabular}

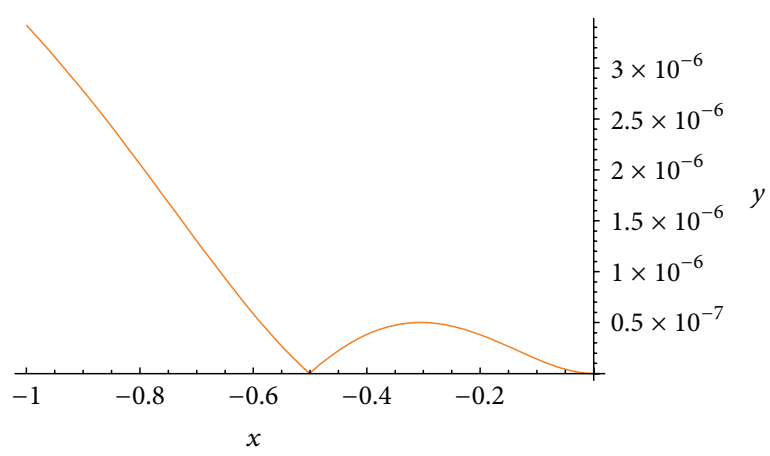

FIGURE 3: Absolute error of the 20th order approximation for $\hbar=$ $-0,368$.

with exact solution $u(x)=e^{x}$ and subjects to initial conditions

$$
u(0)=1, \quad u^{\prime}(0)=1, \quad u^{\prime \prime}(0)=1 .
$$

In this case, we determine the high-order deformation equation (8)-(10) for (18) and (19)

$$
\begin{aligned}
u_{m}(x)=\chi_{m} u_{m-1}(x) & \\
+\hbar \int_{0}^{x} \int_{0}^{\xi} \int_{0}^{\zeta} u_{m-1}^{\prime \prime \prime}(\tau) & -(\tau-1) u_{m-1}^{\prime \prime}(\tau)+(\tau-1) u_{m-1}^{\prime}(\tau) \\
& -u_{m-1}^{\prime}(\tau-1)-u_{m-1}(\tau) \\
& -\left(1-\chi_{m}\right)\left(e^{\tau-1}+e \tau^{2}-\frac{1}{e} \tau^{2}-\frac{2}{e} \tau\right) \\
& -\int_{-1}^{1}\left(\tau t-\tau^{2}\right) u(t) d t d \tau d \zeta d \xi
\end{aligned}
$$

Choosing $u_{0}(x)=(1 / 2) x^{2}+x+1$ as initial approximation which satisfies (19), we successively obtain $u_{i}(x), i=$ $1,2,3, \ldots$, by $(20)$, and we can get

$$
\begin{aligned}
u_{1}(x)= & \frac{\hbar}{e}-\hbar e^{x-1}+\frac{\hbar x}{e}+\frac{\hbar x^{2}}{2 e}-\frac{\hbar x^{3}}{6}-\frac{5 \hbar x^{4}}{72}+\frac{\hbar x^{4}}{12 e} \\
& +\frac{17 \hbar x^{5}}{360}+\frac{\hbar x^{5}}{60 e}-\frac{\hbar e x^{5}}{60}
\end{aligned}
$$

TABLE 3: Comparison of the exact solution and approximations of (18).

\begin{tabular}{lcccc}
\hline$x$ & $U_{\text {exact }}(x)$ & $U_{20}(x, \hbar)$ & $U_{15}(x, \hbar)$ & $U_{10}(x, \hbar)$ \\
\hline-1.0 & 0.3678794 & 0.3678819 & 0.3678847 & 0.3679715 \\
-0.8 & 0.4493289 & 0.4493295 & 0.4493312 & 0.4493805 \\
-0.6 & 0.5488116 & 0.5488117 & 0.5488126 & 0.5488364 \\
-0.4 & 0.6703200 & 0.6703200 & 0.6703203 & 0.6703286 \\
-0.2 & 0.8187307 & 0.8187307 & 0.8187308 & 0.8187320 \\
0.0 & 1.0000000 & 1.0000000 & 1.0000000 & 1.0000000 \\
0.2 & 1.2214027 & 1.2214027 & 1.2214026 & 1.2214008 \\
0.4 & 1.4918246 & 1.4918246 & 1.4918236 & 1.4918054 \\
0.6 & 1.8221188 & 1.8221185 & 1.8221138 & 1.8220361 \\
0.8 & 2.2255409 & 2.2255399 & 2.2255246 & 2.2252881 \\
1.0 & 2.7182818 & 2.7182787 & 2.7182364 & 2.7176359 \\
\hline
\end{tabular}

TABLE 4: The absolute errors approximations of (18).

\begin{tabular}{lccc}
\hline$x$ & Error $_{20}(x, \hbar)$ & Error $_{15}(x, \hbar)$ & Error $_{10}(x, \hbar)$ \\
\hline-1.0 & $2.521916801 E-6$ & $5.306241710 E-6$ & $9214864596 E-5$ \\
-0.8 & $5.731455795 E-7$ & $2.249388334 E-6$ & $5154805044 E-5$ \\
-0.6 & $7.668942051 E-8$ & $9.731825832 E-7$ & $2478243420 E-5$ \\
-0.4 & $3.473543568 E-10$ & $3.435944768 E-7$ & $8.598958233 E-6$ \\
-0.2 & $6.664664611 E-10$ & $5.500709508 E-8$ & $1.283695338 E-6$ \\
0.0 & 0 & 0 & 0 \\
0.2 & $1.655104563 E-9$ & $9.720163109 E-8$ & $1.922279298 E-6$ \\
0.4 & $3.497425126 E-8$ & $1.055423516 E-6$ & $1926022258 E-5$ \\
0.6 & $2.289712260 E-7$ & $4.907220971 E-6$ & $8265793539 E-5$ \\
0.8 & $9.446383729 E-7$ & $1629454606 E-5$ & $2528135763 E-4$ \\
1.0 & $3.079689774 E-6$ & $4536660390 E-5$ & $6458774054 E-4$ \\
\hline
\end{tabular}

$$
\begin{aligned}
U_{m}(x, \hbar) & =\sum_{n=0}^{m} u_{n}(x) \\
& =u_{0}(x)+u_{1}(x)+u_{2}(x)+\cdots+u_{m}(x)
\end{aligned}
$$

The $\hbar$ curves of $U_{20}^{\prime}(1, \hbar)$ and $U_{20}^{\prime \prime}(1, \hbar)$ have been shown in Figure 4 , and the valid region of $\hbar$ is about $-0,7<\hbar<-0,2$.

Finally, we have solutions of (15) and (18) for $\hbar=-0,37$ as shown in Table 3 , and the absolute errors of $U_{20}(x, \hbar)$, $U_{15}(x, \hbar)$, and $U_{10}(x, \hbar)$ for $\hbar=-0,37$ are given in Table 4 . We also give the graphs of the exact solution and 20th order approximation for $\hbar=-0,37$ in Figure 5 and the absolute error of the 20th order approximation for $\hbar=-0,37$ in Figure 6 .

Example 4. Finally, consider the third-order nonlinear integrodifferential-difference equation [9],

$$
\begin{aligned}
& u^{\prime \prime \prime}(x)+\frac{1}{2} u^{\prime \prime}(x)+x u^{\prime}(x)+2 u^{\prime}(x-1)+\frac{1}{2} x u(x) \\
& +u(x-1)=e+\int_{-1}^{0} t u^{2}(t-1) d t
\end{aligned}
$$




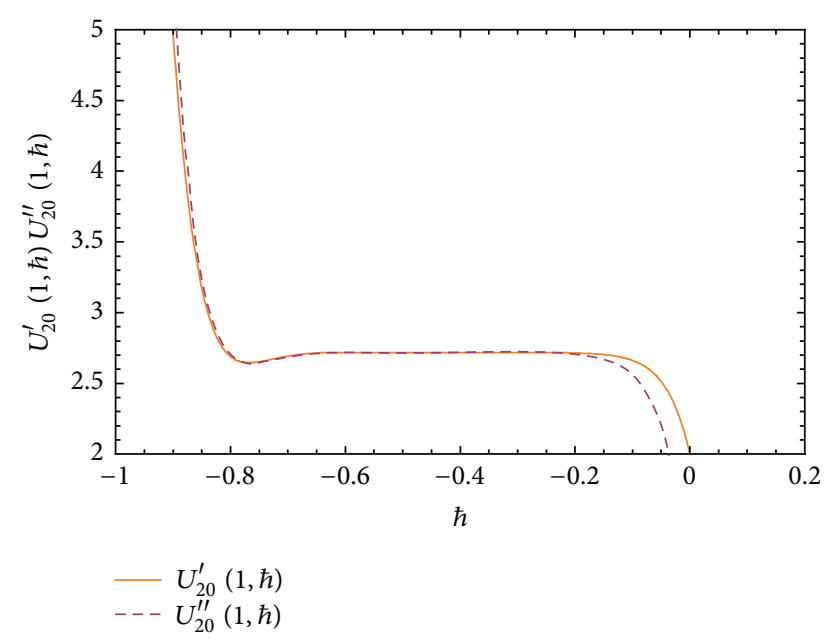

Figure 4: The $\hbar$ curves of $U_{20}^{\prime}(1, \hbar)$ and $U_{20}^{\prime \prime}(1, \hbar)$.

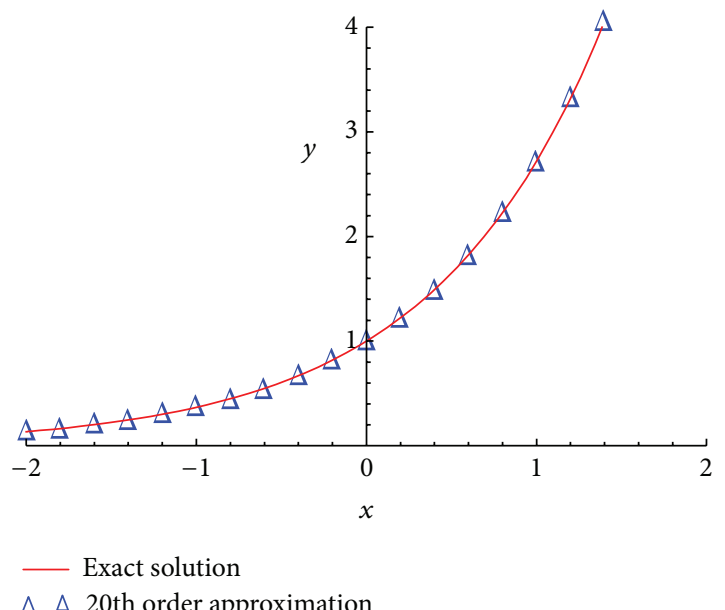

FIGURE 5: Exact solution and 20th order approximation for $\hbar=-0,37$.

subject to initial conditions

$$
u(0)=1, \quad u^{\prime}(0)=-\frac{1}{2}, \quad u^{\prime \prime}(0)=\frac{1}{4}
$$

with exact solution $u(x)=e^{-x / 2}$. Now, the high-order deformation equation (8)-(10) for (22) and (23) is

$$
\begin{aligned}
u_{m}(x)=\chi_{m} u_{m-1}(x) & \\
+\hbar \int_{0}^{x} \int_{0}^{\xi} \int_{0}^{\zeta} & u_{m-1}^{\prime \prime \prime}(\tau)+\frac{1}{2} u_{m-1}^{\prime \prime}(\tau) \\
& +\tau u_{m-1}^{\prime}(\tau)+2 u_{m-1}^{\prime}(\tau-1) \\
& +\frac{1}{2} \tau u_{m-1}(\tau)+u_{m-1}(\tau-1) \\
& -\left(1-\chi_{m}\right) e \\
& -\int_{-1}^{0} t \sum_{i=0}^{m-1} u_{i}(t-1) u_{m-1-i} \\
& \times(t-1) d t d \tau d \zeta d \xi .
\end{aligned}
$$

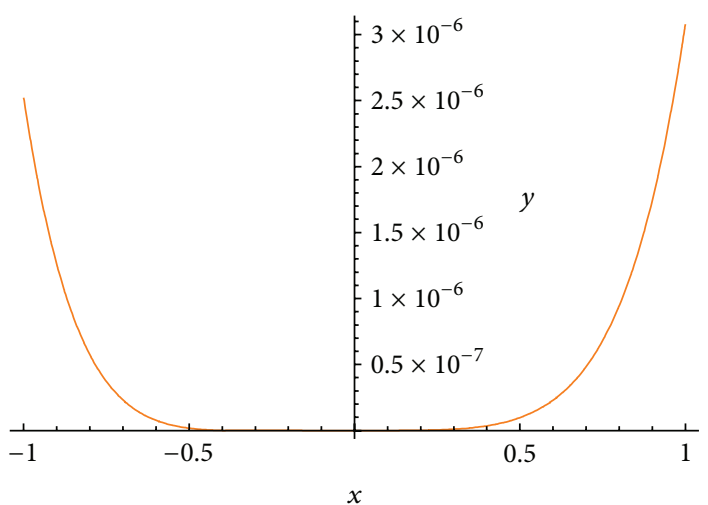

FIgURE 6: Absolute error of the 20th order approximation for $\hbar=$ $-0,37$.

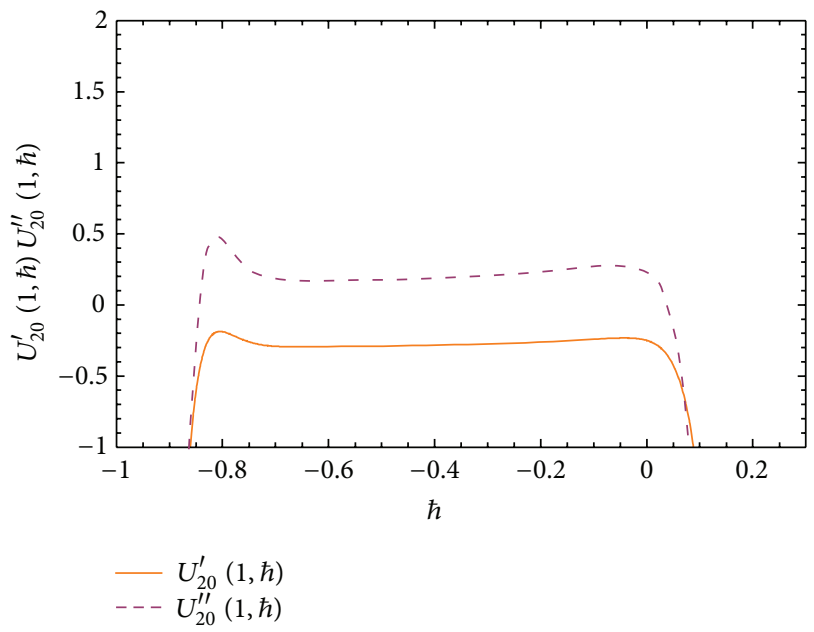

FIGURE 7: The $\hbar$ graphic of $U_{20}^{\prime}(1, \hbar)$ and $U_{20}^{\prime \prime}(1, \hbar)$.

We choose $u_{0}(x)=(1 / 2) x^{2}-x+1$ as initial guess which satisfies (23) and we get

$$
\begin{aligned}
u_{1}(x)= & \hbar\left[\frac{5117}{11520} x^{3}-\frac{1}{6} e x^{3}-\frac{1}{96} x^{4}+\frac{1}{480} x^{5}+\frac{1}{1920} x^{6}\right] \\
& \vdots
\end{aligned}
$$

In Figure 7 , the $\hbar$ curves of $U_{20}^{\prime}(1, \hbar)$ and $U_{20}^{\prime \prime}(1, \hbar)$ have been plotted, and the valid region of $\hbar$ is determined as $-0,7<\hbar<$ 0 .

Then, we also have solutions of (22) for $\hbar=-0,64$ as shown in Table 5 , and the absolute errors of $U_{20}(x, \hbar)$, $U_{15}(x, \hbar)$ and $U_{10}(x, \hbar)$, for $\hbar=-0,64$ are given in Table 6. We also give the graphs of the exact solution and 20th order approximation for $\hbar=-0,64$ in Figure 8 and the absolute error of the 20th order approximation for $\hbar=-0,64$ in Figure 9 . 


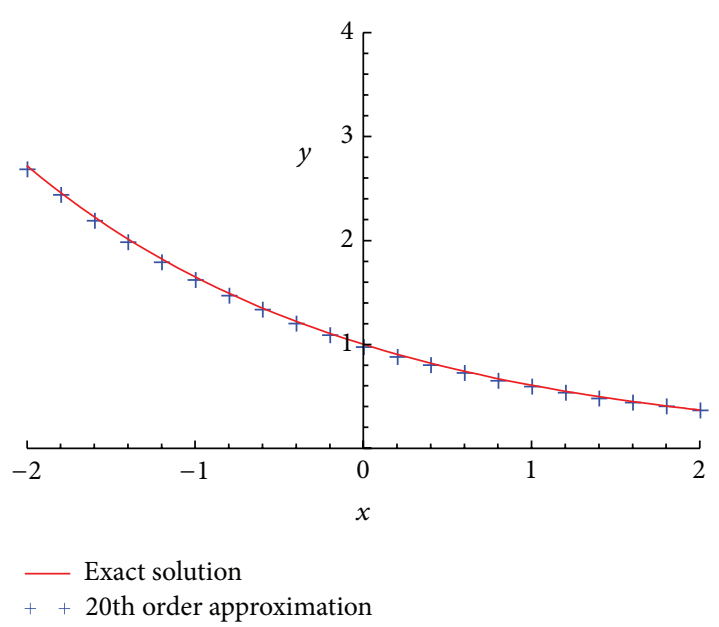

FIGURE 8: Exact solution and 20th order approximation for $\hbar=$ $-0,64$.

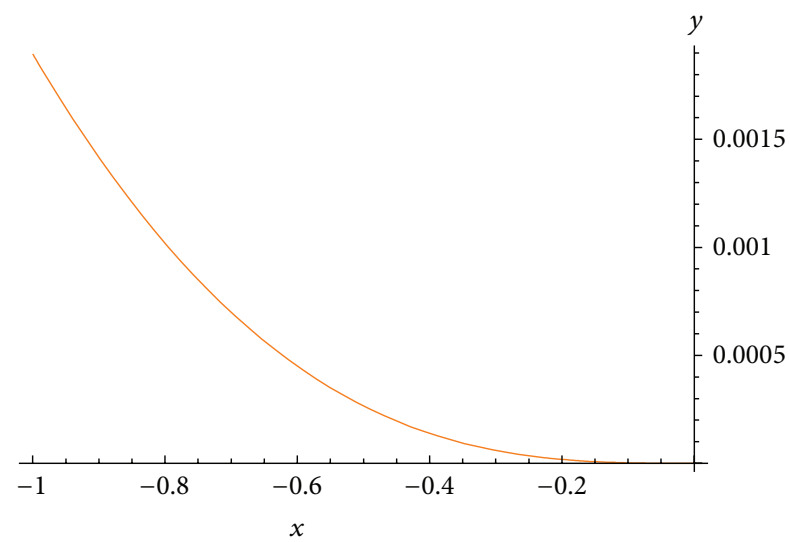

FIGURE 9: Absolute error of the 20th order approximation for $\hbar=$ $-0,64$.

TABLE 5: Comparison of the exact solution and approximations of (22).

\begin{tabular}{lcccc}
\hline$x$ & $U_{\text {exact }}(x)$ & $U_{20}(x, \hbar)$ & $U_{15}(x, \hbar)$ & $U_{10}(x, \hbar)$ \\
\hline-1.0 & 1.6487212 & 1.6468259 & 1.6446986 & 1.6419742 \\
-0.8 & 1.4918246 & 1.4908049 & 1.4896912 & 1.4882483 \\
-0.6 & 1.3498588 & 1.3494086 & 1.3489259 & 1.3482953 \\
-0.4 & 1.2214027 & 1.2212638 & 1.2211163 & 1.2209227 \\
-0.2 & 1.1051709 & 1.1051529 & 1.1051338 & 1.1051088 \\
0.0 & 1.0000000 & 1.0000000 & 1.0000000 & 1.0000000 \\
\hline
\end{tabular}

\section{Conclusions}

In this work, a reliable algorithm based on the HAM to solve various integrodifferential-difference equations with constant and variable coefficients is presented. Some examples are given to illustrate the validity and accuracy of this procedure. The series solutions of (12), (18), and (22) obtained by HAM contain the auxiliary parameter $\hbar$. In general, by means of the so-called $\hbar$ curve, it is straightforward to choose a proper value of $\hbar$ which ensures that the series solution is
TABLE 6: The absolute errors approximations of (22).

\begin{tabular}{lccc}
\hline$x$ & Error $_{20}(x, \hbar)$ & Error $_{15}(x, \hbar)$ & Error $_{10}(x, \hbar)$ \\
\hline-1.0 & $1.8953011 E-3$ & $4.0226668 E-3$ & $6.7470522 E-3$ \\
-0.8 & $1.0197887 E-3$ & $2.1334408 E-3$ & $3.5763888 E-3$ \\
-0.6 & $4.5015457 E-4$ & $9.3282751 E-4$ & $1.5634864 E-3$ \\
-0.4 & $1.3889249 E-4$ & $2.8636722 E-4$ & $4.8001880 E-4$ \\
-0.2 & $1.7997564 E-5$ & $3.7038237 E-5$ & $6.2100619 E-5$ \\
0.0 & 0 & 0 & 0 \\
\hline
\end{tabular}

convergent. Figures 1,4 , and 7 show the $\hbar$ curves obtained from the $m$ th-order HAM approximation solutions. From these figures, the valid regions of $\hbar$ correspond to the line segments nearly parallel to the horizontal axis. Finally, it can be concluded that the homotopy analysis method is a promising tool for both linear and nonlinear IDDEs.

\section{References}

[1] M. Gülsu and Y. Öztürk, "A new collocation method for solution of mixed linear integro-differential-difference equations," Applied Mathematics and Computation, vol. 216, no. 7, pp. 21832198, 2010.

[2] M. Gülsu and M. Sezer, "Approximations to the solution of linear Fredholm integrodifferential-difference equation of high order," Journal of the Franklin Institute, vol. 343, no. 7, pp. 720737, 2006.

[3] K. Maleknejad and Y. Mahmoudi, "Numerical solution of linear Fredholm integral equation by using hybrid Taylor and blockpulse functions," Applied Mathematics and Computation, vol. 149, no. 3, pp. 799-806, 2004.

[4] A. Saadatmandi and M. Dehghan, "Numerical solution of the higher-order linear Fredholm integro-differential-difference equation with variable coefficients," Computers \& Mathematics with Applications, vol. 59, no. 8, pp. 2996-3004, 2010.

[5] N. şahin, S. Yüzbaşı, and M. Sezer, "A Bessel polynomial approach for solving general linear Fredholm integrodifferential-difference equations," International Journal of Computer Mathematics, vol. 88, no. 14, pp. 3093-3111, 2011.

[6] W. Wang, "An algorithm for solving the high-order nonlinear Volterra-Fredholm integro-differential equation with mechanization," Applied Mathematics and Computation, vol. 172, no. 1, pp. 1-23, 2006.

[7] W. Wang and C. Lin, "A new algorithm for integral of trigonometric functions with mechanization," Applied Mathematics and Computation, vol. 164, no. 1, pp. 71-82, 2005.

[8] M. Dehghan and A. Saadatmandi, "Chebyshev finite difference method for Fredholm integro-differential equation," International Journal of Computer Mathematics, vol. 85, no. 1, pp. 123130, 2008.

[9] J. Biazar and M. Gholami Porshokouhi, "Application of variational iteration method for linear and nonlinear integrodifferential-difference equations," International Mathematical Forum, vol. 5, no. 65-68, pp. 3335-3341, 2010.

[10] S. Liao, Beyond Perturbation: Introduction to the Homotopy Analysis Method, vol. 2 of Modern Mechanics and Mathematics, CRC press, Boca Raton, Fla, USA, 2004.

[11] S. J. Liao, Proposed homotopy analysis techniques for the solution of nonlinear problems [Ph.D. thesis], Dissertion, Shanghai Jiao Tong University, 1992. 
[12] S. J. Liao, "Homotopy analysis method: a new analytic method for nonlinear problems," Applied Mathematics and Mechanics, vol. 19, no. 10, pp. 885-890, 1998 .

[13] S. J. Liao, "A uniformly valid analytic solution of twodimensional viscous flow over a semi-infinite flat plate," Journal of Fluid Mechanics, vol. 385, pp. 101-128, 1999.

[14] S. Liao, "An explicit analytic solution to the Thomas-Fermi equation," Applied Mathematics and Computation, vol. 144, no. 2-3, pp. 495-506, 2003.

[15] S. Liao, "On the homotopy analysis method for nonlinear problems," Applied Mathematics and Computation, vol. 147, no. 2, pp. 499-513, 2004.

[16] T. Hayat, T. Javed, and M. Sajid, "Analytic solution for rotating flow and heat transfer analysis of a third-grade fluid," Acta Mechanica, vol. 191, no. 3-4, pp. 219-229, 2007.

[17] T. Hayat, M. Khan, M. Sajid, and S. Asghar, "Rotating flow of a third grade fluid in a porous space with hall current," Nonlinear Dynamics, vol. 49, no. 1-2, pp. 83-91, 2007.

[18] S. Abbasbandy, "Homotopy analysis method for heat radiation equations," International Communications in Heat and Mass Transfer, vol. 34, no. 3, pp. 380-387, 2007.

[19] S. Abbasbandy and E. Shivanian, "A new analytical technique to solve Fredholm's integral equations," Numerical Algorithms, vol. 56, no. 1, pp. 27-43, 2011.

[20] S. Abbasbandy and F. S. Zakaria, "Soliton solutions for the 5th-order KdV equation with the homotopy analysis method," Nonlinear Dynamics, vol. 51, no. 1-2, pp. 83-87, 2008.

[21] Z. Wang, L. Zou, and H. Zhang, "Applying homotopy analysis method for solving differential difference equation," Physics Letters A, vol. 369, no. 1-2, pp. 77-84, 2007.

[22] A. S. Bataineh, M. S. M. Noorani, and I. Hashim, "Solutions of time-dependent Emden-Fowler type equations by homotopy analysis method," Physics Letters A, vol. 371, no. 1-2, pp. 72-82, 2007.

[23] M. Inc, "On exact solution of laplace equation with dirichlet and neumann boundary conditions by the homotopy analysis method," Physics Letters A, vol. 365, no. 5-6, pp. 412-415, 2007.

[24] F. Awawdeh, A. Adawi, and S. Al-Shara, "Analytic solution of multipantograph equation," Journal of Applied Mathematics and Decision Sciences, vol. 2008, Article ID 605064, 11 pages, 2008. 


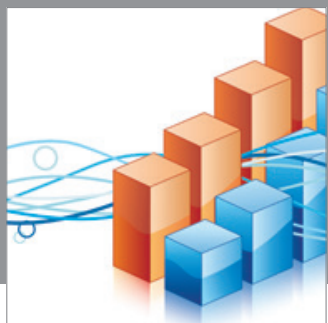

Advances in

Operations Research

mansans

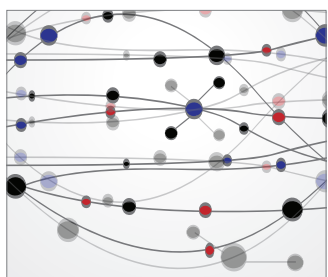

The Scientific World Journal
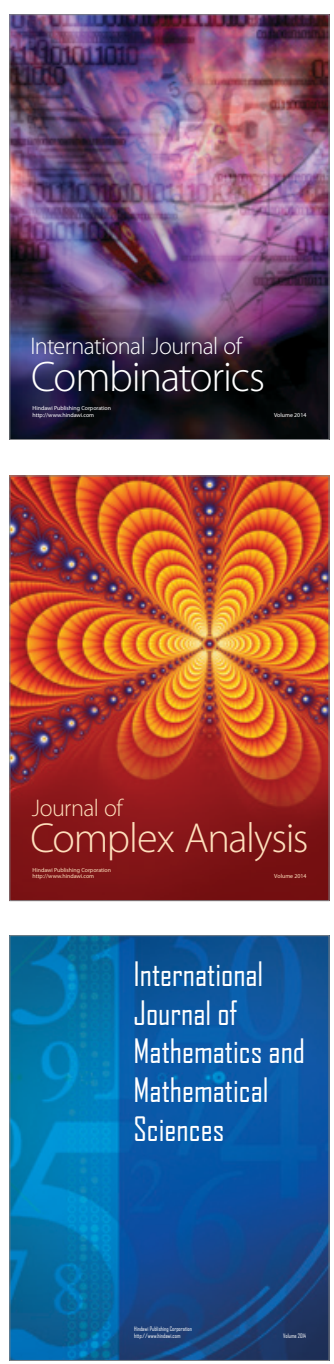
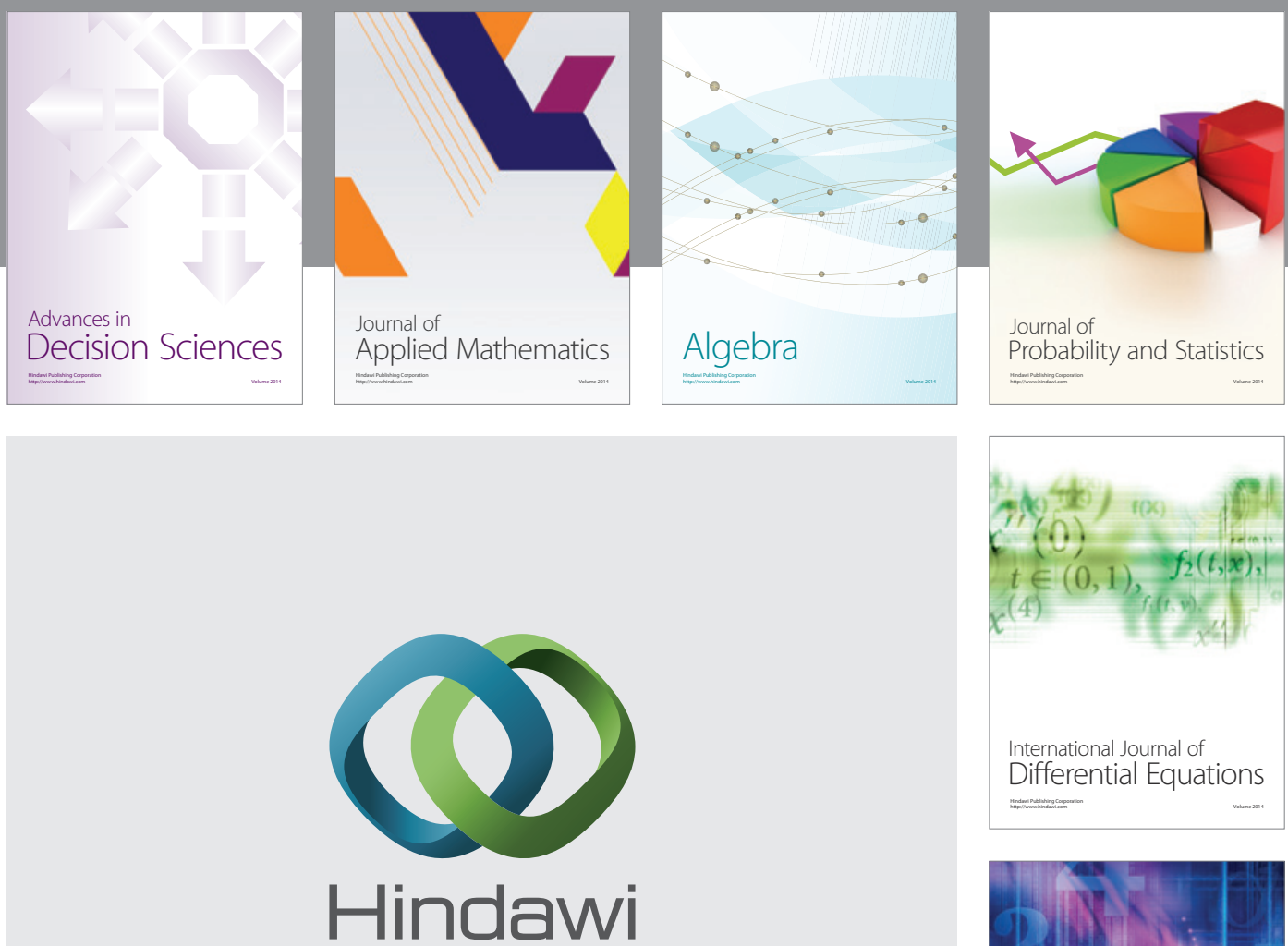

Submit your manuscripts at http://www.hindawi.com
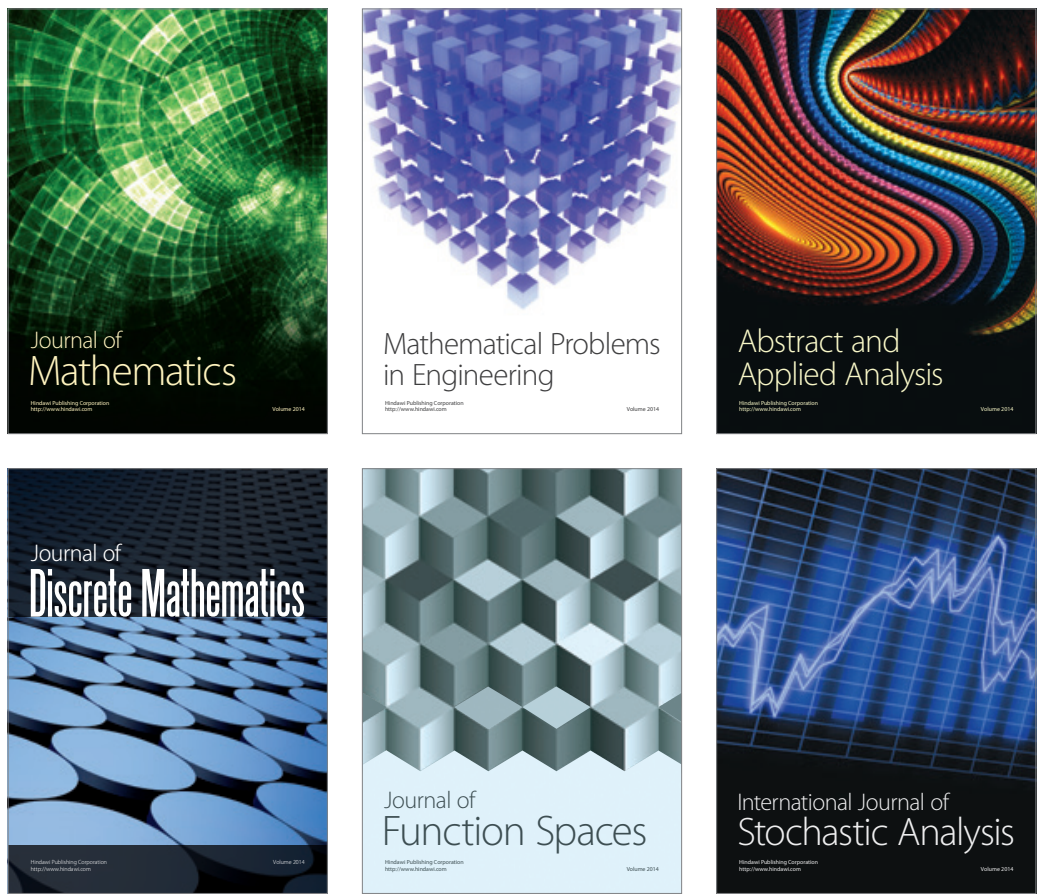

Journal of

Function Spaces

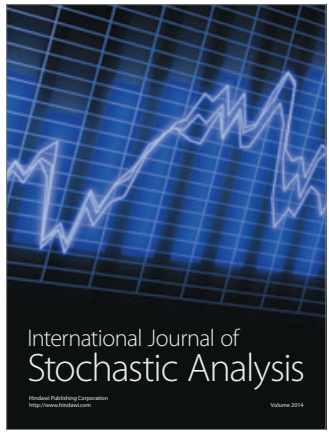

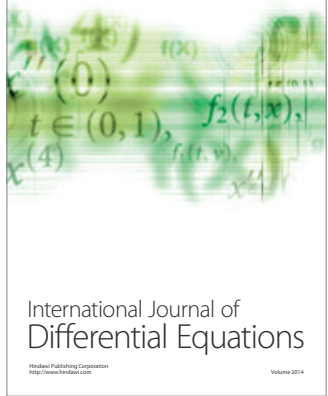
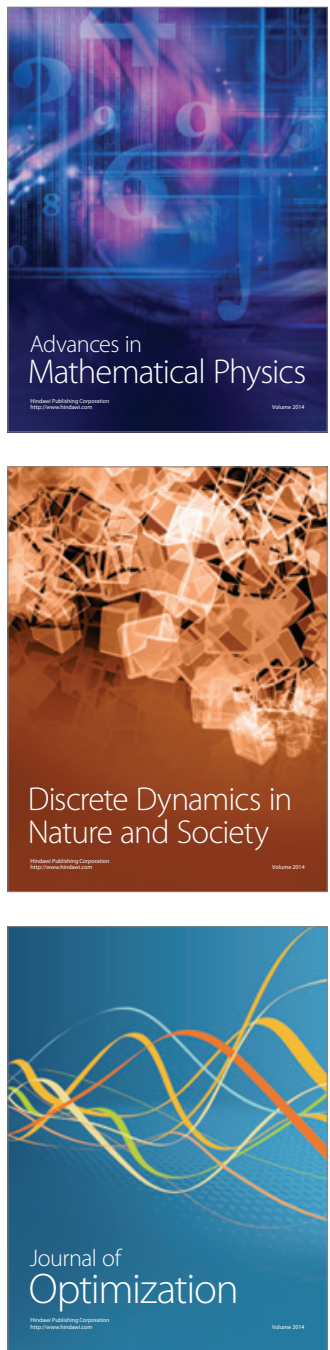